\title{
High-power Q-switched laser with high-order Laguerre-Gaussian modes: application for extra-cavity harmonic generations
}

\author{
Y.J. Huang • P.Y. Chiang $\cdot$ H.C. Liang $\cdot$ K.W. Su • \\ Y.F. Chen
}

Received: 14 January 2011 / Revised version: 14 February 2011 / Published online: 15 April 2011

(C) Springer-Verlag 2011

\begin{abstract}
We present a compact efficient scheme for generation of high-order Laguerre-Gaussian $\mathrm{TEM}_{p, 0}$ modes in a diode-pumped actively Q-switched $\mathrm{Nd}^{\mathrm{YVO}} \mathrm{YV}_{4}$ laser. The resonator is composed of two plane mirrors with an intracavity concave lens to expand the cavity mode size for generating a high-power Laguerre-Gaussian $\mathrm{TEM}_{5,0}$ mode. We further confirm that the $\mathrm{TEM}_{5,0}$ mode is noticeably superior to the $\mathrm{TEM}_{0,0}$ mode in the processes of second and third harmonic generations.
\end{abstract}

\section{Introduction}

In recent years, ultraviolet (UV) light sources have rapidly attracted a lot of interest because they are useful in numerous applications such as rapid prototyping, laser printing, laser processing, spectroscopy, optical data storage, medical treatment and so on. Compared with other UV lasers, diode-pumped all-solid-state lasers with extra-cavity nonlinear frequency conversion intrinsically possess advantages of smaller focused size, higher efficiency, longer life time, higher stability, easier implementation and smaller system size, etc. [1, 2]. Currently, the fundamental Gaussian modes are usually used in the processes of second and third harmonic generations (SHG and THG) because of the good spatial property [3, 4].

In cylindrical coordinates, the transverse modes can be expressed in terms of Laguerre-Gaussian modes with the la-

Y.J. Huang · P.Y. Chiang · H.C. Liang · K.W. Su · Y.F. Chen $(\varangle)$ Department of Electrophysics, National Chiao Tung University, 1001 TA Hsueh Road, Hsinchu 30050, Taiwan

e-mail: yfchen@cc.nctu.edu.tw

Fax: +886-35-725230 bel of $\operatorname{TEM}_{p, l}$, where $p$ and $l$ represent the number of radial nodes and azimuthal nodes, respectively. In the past years, several investigations have been carried out to generate lowand higher-order $\mathrm{TEM}_{p, l}$ modes in solid-state lasers [5-11]. Among these studies, $\operatorname{TEM}_{p, l}$ modes with $p \neq 0$ and $l=0$ have been demonstrated $[5,12]$ to be similar to the Bessellike beam that possesses a central peak with a divergence considerably lower than that of the Gaussian beam with the same waist [13-17]. Bessel-like modes have already been utilized as light sources in lithographic patterning, laser machining, and nonlinear optics [18-22]. However, so far highorder Laguerre-Gaussian TEM ${ }_{p, 0}$ modes have not been employed in processes of SHG and THG.

In this work, we originally design a compact threeelement resonator to excite high-order Laguerre-Gaussian $\mathrm{TEM}_{p, 0}$ modes in a diode-pumped actively Q-switched $\mathrm{Nd}: \mathrm{YVO}_{4}$ laser. We use flat-flat cavity with an intra-cavity concave lens to expand the cavity mode size for efficiently generating the Laguerre-Gaussian $\mathrm{TEM}_{5,0}$ mode with the output power up to $8.52 \mathrm{~W}$ at a pulse repetition rate of $40 \mathrm{kHz}$. We experimentally confirm that the conversion efficiencies in the processes of SHG and THG obtained with the $\mathrm{TEM}_{5,0}$ mode are noticeably higher than the results obtained with the $\mathrm{TEM}_{0,0}$ mode. The optical-to-optical conversion efficiencies from $1064 \mathrm{~nm}$ to $355 \mathrm{~nm}$ are found to be $35.6 \%$ and $28.1 \%$ for the $\mathrm{TEM}_{5,0}$ mode and $\mathrm{TEM}_{0,0}$ mode, respectively.

\section{Cavity configuration and analysis}

The radial intensity of the Laguerre-Gaussian $\operatorname{TEM}_{p, l}$ modes is given by 
Fig. 1 Schematic of the cavity setup for a diode-pumped $\mathrm{AO}$ Q-switched $\mathrm{Nd}: \mathrm{YVO}_{4}$ laser

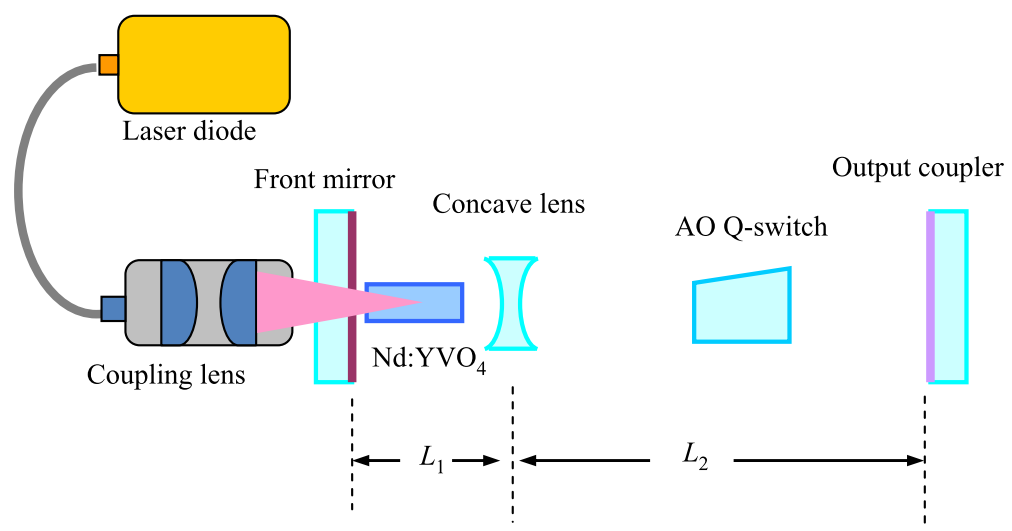

$$
\begin{aligned}
I_{p, l}(\rho, z)= & \frac{2 p !}{\pi(p+|l|) !} \frac{1}{w^{2}(z)}\left(\frac{2 \rho^{2}}{w(z)^{2}}\right)^{l} L_{p}^{l}\left(\frac{2 \rho^{2}}{w(z)^{2}}\right) \\
& \times \exp \left[-\frac{2 \rho^{2}}{w(z)^{2}}\right],
\end{aligned}
$$

where $w(z)=w_{0} \sqrt{1+\left(z / z_{R}\right)^{2}}, w_{0}$ is the beam radius at the waist, and $z_{R}=\pi w_{0}^{2} / \lambda$ is the Rayleigh range, and $L_{p}^{l}(\cdot)$ are the associated Laguerre polynomials. Flood et al. [5] demonstrated that high-order Laguerre-Gaussian $\mathrm{TEM}_{p, 0}$ modes could be generated in an end-pumped solid-state laser when the pump spot size $\omega_{p}$ was considerably smaller than the fundamental $\mathrm{TEM}_{0,0}$-mode spot size $\omega_{0}$ of the cavity, i.e., $\omega_{o} / \omega_{p} \gg 1$. Although the pump beams can be tightly focused to reach the criterion of $\omega_{o} / \omega_{p} \gg 1$, the tight focusing of pump beam inevitably causes strong thermal effects such as thermal lensing, thermal fracture, etc., leading to the obstruction for the power scale-up. Therefore, a practical way is to expand the cavity mode size without tight focusing of the pump beam. Here, we design a compact efficient three-element resonator, consisting of a flat front mirror, a concave lens and a flat output coupler, to obtain large fundamental mode sizes.

Figure 1 shows the configuration of the three-element cavity for an end-pumped acousto-optic (AO) solid-state laser, where $L_{1}$ is the distance between the front mirror and the concave lens, $L_{2}$ is the distance between the concave lens and the flat output coupler, and $f$ is the focal length of the concave lens. The flat front mirror was antireflection (AR) coated at $808 \mathrm{~nm}$ on the entrance face and was coated at $808 \mathrm{~nm}$ for high transmission as well as $1064 \mathrm{~nm}$ for high reflection (HR) on the second surface. The gain medium was a 0.1 at. $\% \mathrm{Nd}: \mathrm{YVO}_{4}$ crystal with dimensions of $3 \times 3 \times 12 \mathrm{~mm}^{3}$ and was located to be adjacent the front mirror for convenience of end-pumping scheme. Both facets of the laser crystal were AR coated at $808 \mathrm{~nm}$ and $1064 \mathrm{~nm}$. The laser crystal was wrapped with indium foil and mounted in a water-cooled copper heat sink at $20^{\circ} \mathrm{C}$. Although using the laser crystal with the HR-AR coating is preferable for more compact configuration, we use a separate front mirror and the AR-AR coated laser crystal in the present setup because of the availability of experimental components. A concave lens with AR coating at $1064 \mathrm{~nm}$ on both faces was placed just behind the laser crystal. A 20-mmlong AO Q-switch (NEOS technologies) with AR coating at $1064 \mathrm{~nm}$ on both faces was placed in the center of the cavity and was driven at a central frequency of $41 \mathrm{MHz}$ with RF power of $25 \mathrm{~W}$. The pump source was a $30-\mathrm{W} 808-\mathrm{nm}$ fiber-coupled laser diode with a core diameter of $600 \mu \mathrm{m}$ and a numerical aperture of 0.16 . The pump beam was reimaged into the laser crystal with a lens set that has the focal length of $25 \mathrm{~mm}$ with a magnification of unity and the coupling efficiency of $90 \%$. As mentioned earlier, the use of the relatively large pump radius is beneficial for power scaling with the avoidance of serious thermal problems. The flat output coupler with $50 \%$ transmission was employed during the experiment. For constructing a compact Q-switched laser, we set the optical cavity length to be $L_{1}=20 \mathrm{~mm}$ and $L_{2}=120 \mathrm{~mm}$.

The effective focal length of the thermal lens in a $\mathrm{Nd}: \mathrm{YVO}_{4}$ crystal could be expressed as [23]:

$$
\begin{aligned}
\frac{1}{f_{\mathrm{th}}}= & \frac{\xi}{\pi K_{c}} \int_{0}^{l} \frac{\alpha e^{-\alpha z}}{1-e^{-\alpha l}} \frac{1}{\omega_{p}^{2}(z)} \\
& \times\left[\frac{1}{2} \frac{d n}{d T}+(n-1) \alpha_{T} \omega_{p}(z) / l\right] d z,
\end{aligned}
$$

where $\xi$ is the fraction of pump power that results in heat, $K_{c}$ is the thermal conductivity of the laser material, $\alpha$ is the absorption coefficient, $n$ is the refractive index, $l$ is the length of the gain medium, $d n / d T$ is the thermal-optic coefficient, $\alpha_{T}$ is the thermal expansion coefficient, and $\omega_{p}(z)$ is the variation of the pump radius. With the following parameters: $K_{c}=5.23 \mathrm{~W} / \mathrm{m} \mathrm{K}, \alpha=0.2 \mathrm{~mm}^{-1}, n=2.1652, l=12 \mathrm{~mm}$, $d n / d T=3 \times 10^{-6} \mathrm{~K}^{-1}, \alpha_{T}=4.43 \times 10^{-6} \mathrm{~K}^{-1}$, the effective focal length of thermal lens was estimated to be approximately $f_{\text {th }}=220 \mathrm{~mm}$ at a pump power of $25 \mathrm{~W}$. We then use the ABCD-matrix method to calculate the cavity mode size at the front mirror as a function of the effective 
thermal focal length for a given focal length of the concave lens. Figure 2 depicts the calculated results for the four cases of $f=-200 \mathrm{~mm},-300 \mathrm{~mm},-400 \mathrm{~mm}$, and $-\infty$. Note that the case of $f=-\infty$ means the cavity without the concave lens. As seen in Fig. 2, the cavity mode size without a concave lens is approximately equal to the pump spot size. Although a concave lens can effectively enlarge the cavity mode size in some regime, the cavity needs an appropriate thermal focal length to step into the stable region. With the $\mathrm{ABCD}$-matrix theory, we can find that the stable region is given by

$$
\begin{aligned}
& \left(L_{1}-f\right)\left[1-\frac{f^{2}}{f^{2}+L_{1} L_{2}-\left(L_{1}+L_{2}\right) f}\right] \\
& \leq f_{\text {th }} \leq\left(L_{1}-f\right) .
\end{aligned}
$$

The thermal focal length in the present setup approximately changes from $320 \mathrm{~mm}$ to $220 \mathrm{~mm}$ for the pump power in-

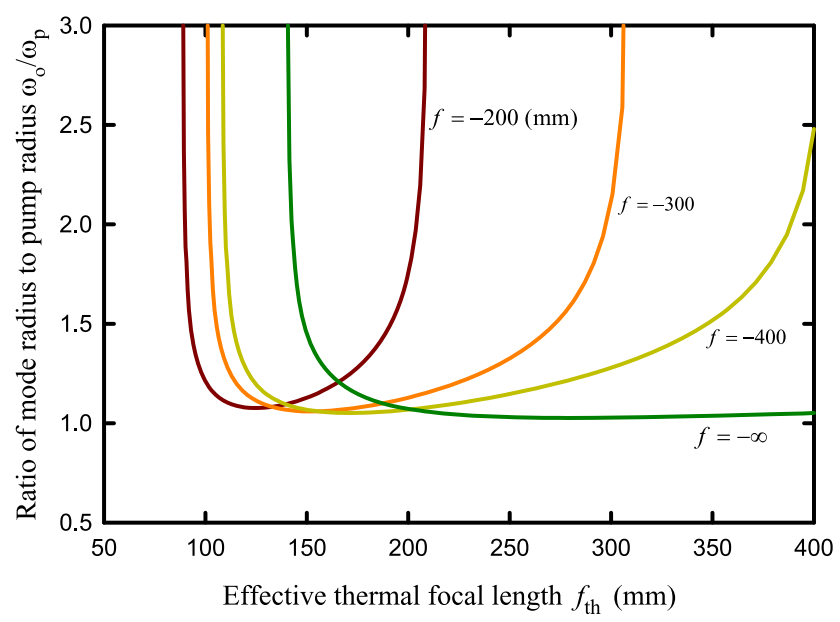

Fig. 2 Calculated results for the ratio of cavity mode size at the front mirror to pump size as a function of the effective thermal focal length for the cases of $f=-200 \mathrm{~mm},-300 \mathrm{~mm},-400 \mathrm{~mm}$, and $-\infty$ creasing from $10 \mathrm{~W}$ to $25 \mathrm{~W}$. As a result, we chose a concave lens with $f=-300 \mathrm{~mm}$ to meet the range of the thermal focal length.

\section{High-order Laguerre-Gaussian $\mathrm{TEM}_{p, 0}$ modes}

First of all, the laser experiment for the standard flat-flat cavity without a concave lens was performed to measure the transverse pattern as a baseline for comparison. To record the transverse profiles of the different structure, the laser output was directly projected on a screen at a distance of approximately $1220 \mathrm{~mm}$ behind the output coupler and the scattered light was captured by a digital camera. As seen in Fig. 3(a), the transverse mode in the resonator without a concave lens is a pure fundamental TEM $\mathrm{T}_{0,0}$ mode. Figure 3(b) depicts the experimental transverse pattern observed in the laser cavity with a concave lens of $f=-300 \mathrm{~mm}$ at a pump power of $25 \mathrm{~W}$. It can be seen that the spatial distribution displays the lasing mode to be dominated by a Laguerre-Gaussian TEM $_{5,0}$ mode with negligible fundamental Gaussian mode. When the pump radius was changed from $300 \mu \mathrm{m}$ to $100 \mu \mathrm{m}$, we found that the dominated transverse mode varied from TEM$_{5,0}$ mode to TEM $_{8,0}$ mode, as shown in Fig. 3(c). In short, we have experimentally confirmed the proportionality between the transverse order and the ratio $\omega_{o} / \omega_{p}$, but the detailed theoretical analysis of the dependence of the transverse order on the ratio $\omega_{o} / \omega_{p}$ is beyond the scope of this work.

As found by Flood et al. [5], we confirmed that the experimental Laguerre-Gaussian $\mathrm{TEM}_{5,0}$ mode has a central peak with a divergence considerably lower than that of a $\mathrm{TEM}_{0,0}$ mode with the same waist. This observation indicates that the experimental TEM $_{5,0}$ mode might be beneficial for the achievement of the higher frequency conversion efficiency in processes of SHG and THG. Before investigating the conversion efficiency in nonlinear optics, we make
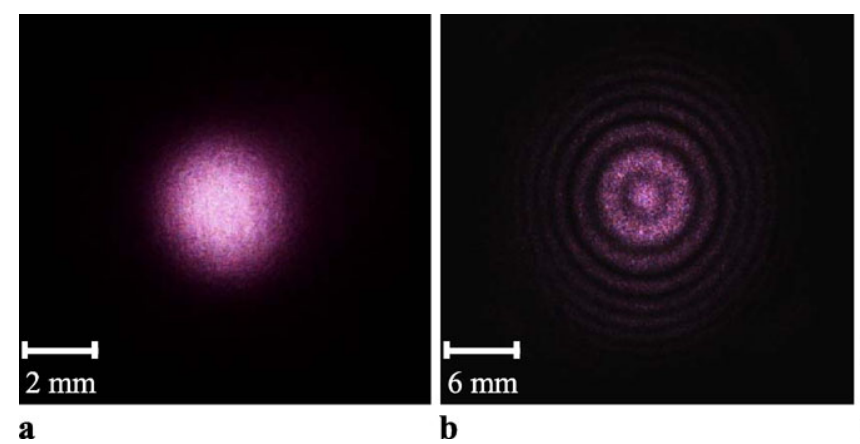

b

Fig. 3 Experimental transverse mode patterns of (a) TEM $_{00}$ mode without a concave lens at a pump power of $25 \mathrm{~W}$; (b) $\mathrm{TEM}_{5,0}$ mode with a concave lens $(f=-300 \mathrm{~mm})$ and a pump radius of $300 \mu \mathrm{m}$ at a pump power of $25 \mathrm{~W}$; (c) $\mathrm{TEM}_{8,0}$ mode with a concave lens
( $f=-300 \mathrm{~mm}$ ) and a pump radius of $100 \mu \mathrm{m}$ at a pump power of $25 \mathrm{~W}$. The patterns were measured at a distance of approximately $1220 \mathrm{~mm}$ behind the output coupler 
a comparison of the laser performances of the cavities with and without the concave lens. Figure 4 shows the average output power as a function of the incident pump power for both configurations operating at a pulse repetition rate of $40 \mathrm{kHz}$. The cavity with the concave lens has a considerably lager pump threshold because the thermal focal power of the crystal should be greater than $\left(L_{1}-f\right)^{-1}$ to result in the cavity to be stable. At a pump power of $25 \mathrm{~W}$, the maximum average output powers at a repetition rate of $40 \mathrm{kHz}$ were $8.52 \mathrm{~W}$ and $8.76 \mathrm{~W}$ for the cavities with and without concave lens. In other words, the difference of the maximum average output power between both cavity configurations was rather small. Figure 5 depicts the dependence of the average output powers, pulse energies and peak powers on the pulse repetition rate at a pump power of $25 \mathrm{~W}$ for both cavity configurations. On the whole, the average output power of the cavity with a concave lens was smaller than that of the cavity without a concave lens by approximately $5-10 \%$, as shown in Fig. 5(a). The pulse temporal behavior was recorded by a LeCroy digital oscilloscope (Wavepro 7100, $10 \mathrm{G}$ samples/s,

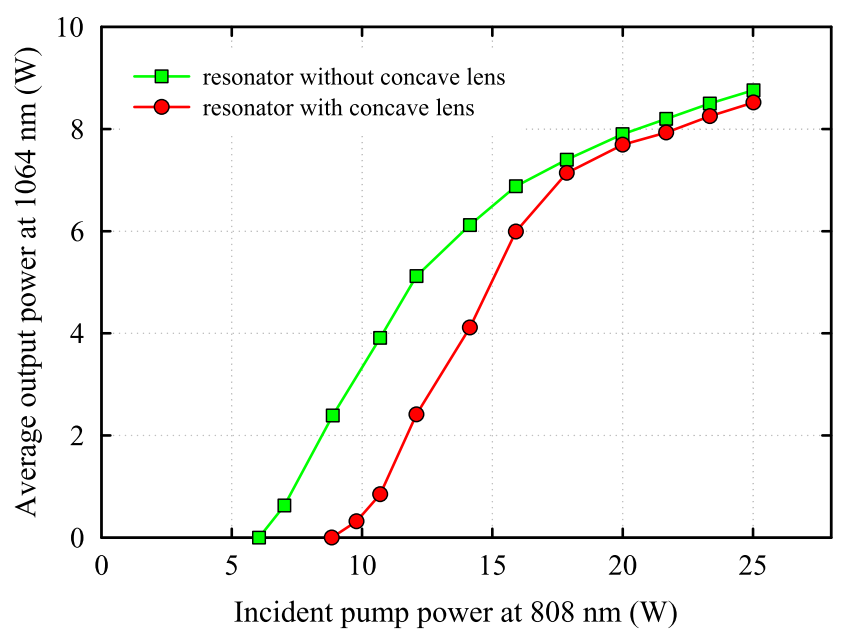

Fig. 4 Average output power at a repetition rate of $40 \mathrm{kHz}$ as a function of the incident pump power for the cavities with and without the concave lens
$1 \mathrm{GHz}$ bandwidth) with a fast Si photodiode. The pulse durations for both configurations were experimentally found nearly the same. With increasing the pulse repetition rate from $20 \mathrm{kHz}$ to $80 \mathrm{kHz}$, the pulse duration was found to vary from 7 to $16 \mathrm{~ns}$. As a result, the pulse energy and the peak power obtained with a concave lens were generally smaller than the results obtained without a concave lens, as shown in Fig. 5(b) and 5(c). In the following section, we make a comparison of the high-order TEM $_{5,0}$ mode and the fundamental $\mathrm{TEM}_{0,0}$ mode in the processes of extra-cavity SHG and THG.

\section{Conversion efficiencies of extra-cavity SHG and THG}

Here lithium triborate (LBO) crystals are exploited as nonlinear frequency converters for SHG and THG since they have the advantages of high damage threshold, relatively large acceptance angle, and small walk-off angle. One LBO crystal with dimensions of $3 \times 3 \times 15 \mathrm{~mm}^{3}$ was cut at $\theta=90^{\circ}, \varphi=10.4^{\circ}$ for type-I phase-matched SHG at temperature of $46.6^{\circ} \mathrm{C}$. Both facets of the SHG crystal were AR coated at $1064 \mathrm{~nm}$ and $532 \mathrm{~nm}$. Another LBO crystal with dimensions of $3 \times 3 \times 10 \mathrm{~mm}^{3}$ was cut at $\theta=44^{\circ}$, $\varphi=90^{\circ}$ for type-II phase-matched THG at temperature of $48^{\circ} \mathrm{C}$. Both facets of the THG crystal were AR coated at $1064 \mathrm{~nm}, 532 \mathrm{~nm}$, and $355 \mathrm{~nm}$. The temperatures of the SHG and THG nonlinear crystals were monitored by thermoelectric controllers with the precision of $0.1^{\circ} \mathrm{C}$. Two convex lenses were used to focus the laser beams into the SHG and THG nonlinear crystals for achieving efficient nonlinear conversion. The former one with focal length of $38 \mathrm{~mm}$ was AR coated at $1064 \mathrm{~nm}$ on both sides, the latter one with focal length of $19 \mathrm{~mm}$ was AR coated at $1064 \mathrm{~nm}$ and $532 \mathrm{~nm}$ on both sides. The optimized geometrical distances of $L_{3}, L_{4}$, $L_{5}$, and $L_{6}$ indicated in Fig. 6 were experimentally found to be approximately $70 \mathrm{~mm}, 30 \mathrm{~mm}, 25 \mathrm{~mm}$, and $30 \mathrm{~mm}$, respectively.
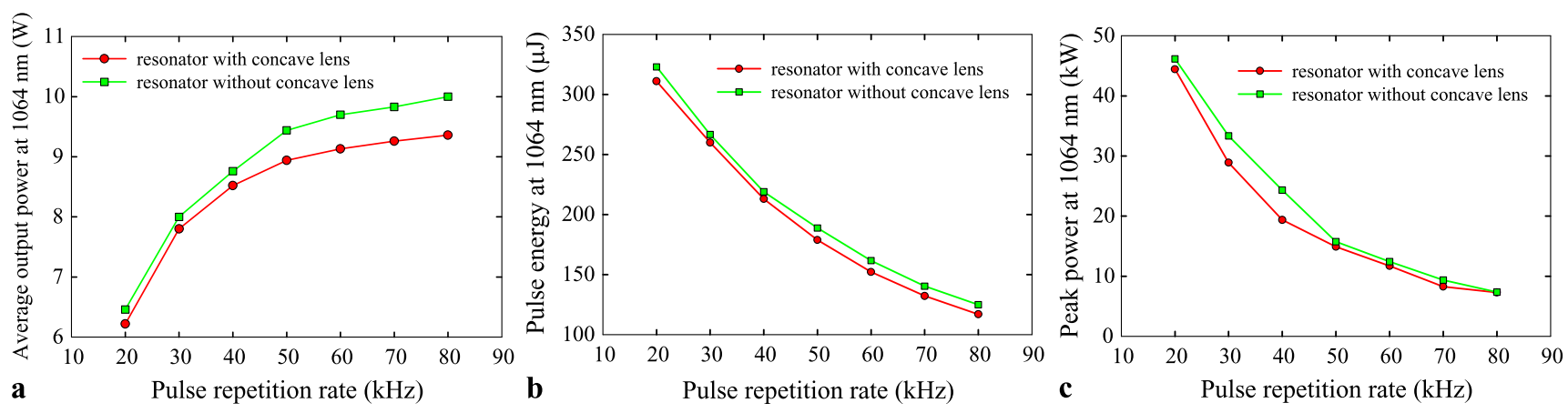

Fig. 5 Dependence of the (a) average output power, (b) pulse energy, and (c) peak power on the pulse repetition rate at a pump power of $25 \mathrm{~W}$ for the cavities with and without the concave lens 
Fig. 6 Schematic of the experimental setup for extra-cavity SHG and THG

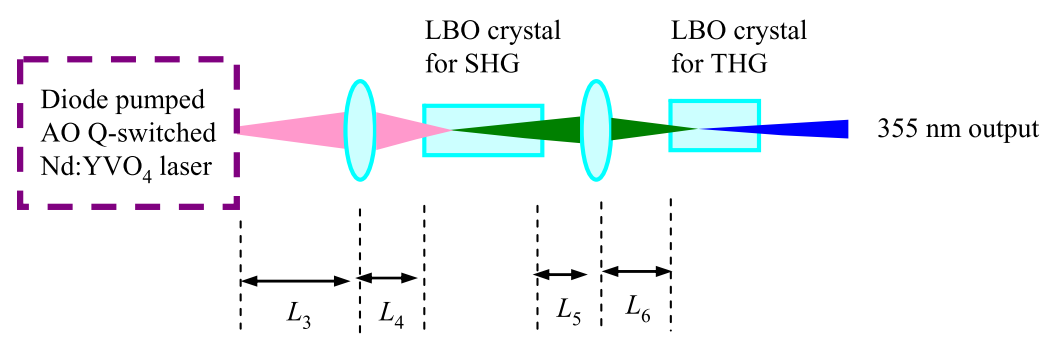

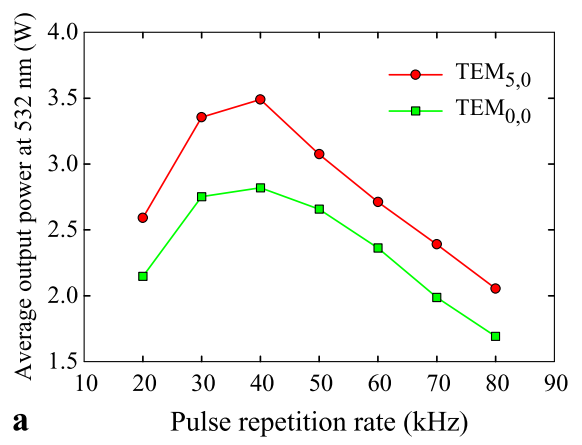
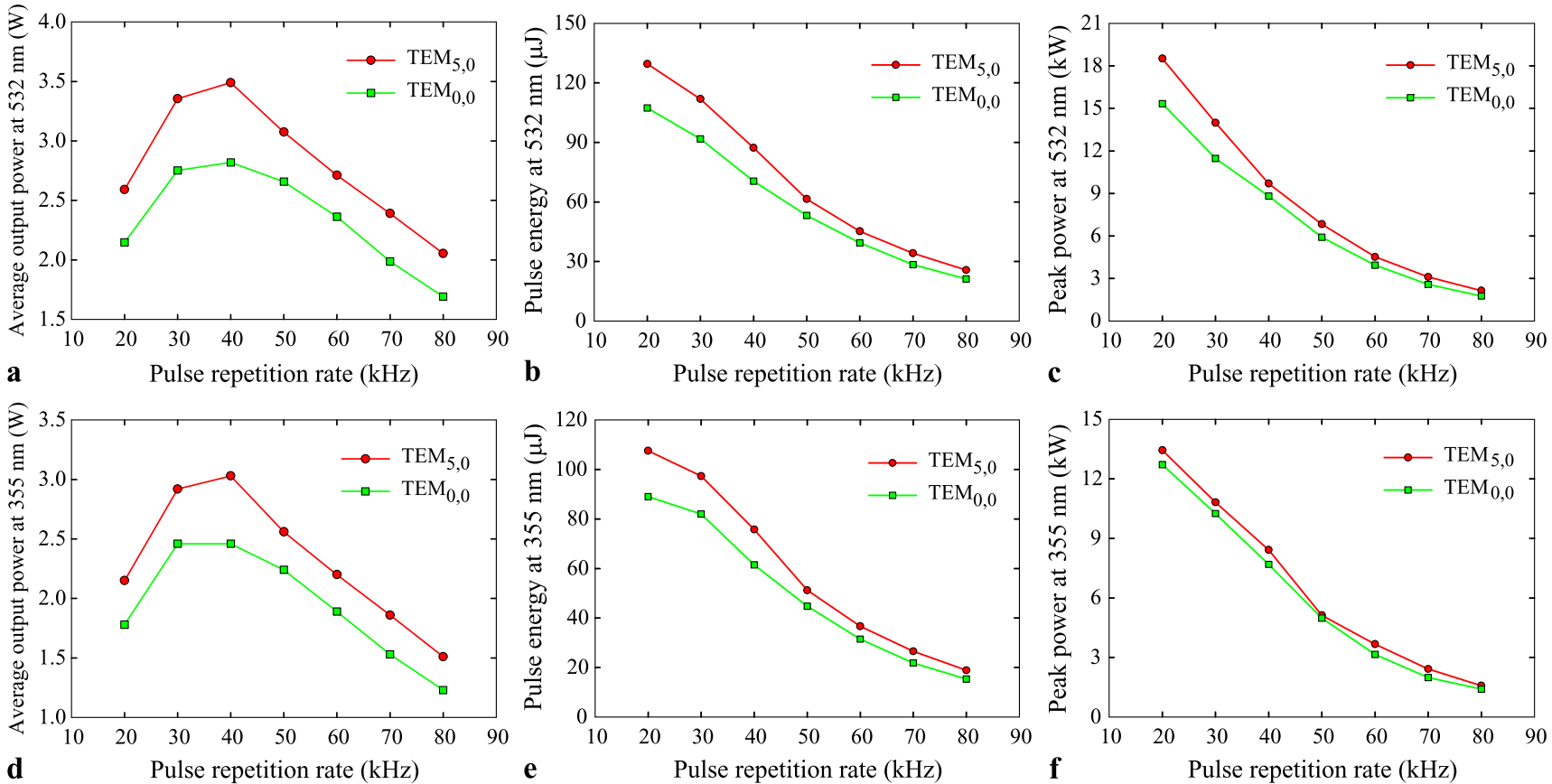

Fig. 7 Harmonic generation performances: (a) average output power, (b) pulse energy, (c) peak power at $532 \mathrm{~nm}$; and (d) average output power, (e) pulse energy, (f) peak power at $355 \mathrm{~nm}$ versus the pulse repetition rate at a pump power of $25 \mathrm{~W}$ for the high-order $\mathrm{TEM}_{5,0}$ mode and the fundamental $\mathrm{TEM}_{0,0}$ mode

the calculated results for the case of $f=-\infty$ in Fig. 2, the mode radius of the $\mathrm{TEM}_{0,0}$ mode is about $300 \mu \mathrm{m}$. A Rayleigh range of such a $\mathrm{TEM}_{0,0}$ beam is estimated to be $26.6 \mathrm{~cm}$, so the divergence of the beam would be rather low. However, we experimentally found that the superiority of the $\mathrm{TEM}_{5,0}$ mode over the fundamental $\mathrm{TEM}_{0,0}$ mode in the processes of extra-cavity SHG and THG. This experimental observation is regard as a result of the considerable narrowness of the central peak of the high-order LaguerreGaussian TEM$_{p, 0}$ mode than that of the Gaussian beam.

It is worthwhile to mention that although the generation of high-order Laguerre-Gaussian $\operatorname{TEM}_{p, 0}$ modes in a Q-switched laser has been reported with the other method [9], our experimental setup intrinsically owns the advantages of compactness, simplicity and is potentially beneficial for power scaling. In comparison with [5], expanding the cavity mode size instead of tight focusing of the pump beam can remarkably relieve the thermal problems under high-power operation. Furthermore, the application and $9.8 \%$ (from $808 \mathrm{~nm}$ to $355 \mathrm{~nm}$ ), respectively. Based on 
of high-order Laguerre-Gaussian modes in the process of extra-cavity SHG and THG was investigated for the first time and we experimentally found the $\mathrm{TEM}_{p, 0}$ mode is noticeably superior to the $\mathrm{TEM}_{0,0}$ mode in the harmonic generation process.

\section{Conclusion}

In summary, we have successfully designed a compact resonator to generate high-power Laguerre-Gaussian $\mathrm{TEM}_{5,0}$ mode. The resonator is composed of two plane mirrors with an intra-cavity concave lens to expand the cavity mode size. At a pump power of $25 \mathrm{~W}$, the average output power for the Laguerre-Gaussian TEM $_{5,0}$ mode was found to be up to $8.52 \mathrm{~W}$ at a pulse repetition rate of $40 \mathrm{kHz}$. Furthermore, we experimentally verify that the $\mathrm{TEM}_{5,0}$ mode is conspicuously superior to the $\mathrm{TEM}_{0,0}$ mode in the processes of SHG and THG. At an incident pump power of $25 \mathrm{~W}$ at $808 \mathrm{~nm}$ and a repetition rate of $40 \mathrm{kHz}$, the maximum output powers at $355 \mathrm{~nm}$ obtained with the $\mathrm{TEM}_{5,0}$ mode and $\mathrm{TEM}_{0,0}$ mode were $3.1 \mathrm{~W}$ and $2.45 \mathrm{~W}$, respectively. The optical-to-optical conversion efficiencies from $1064 \mathrm{~nm}$ to $355 \mathrm{~nm}$ were found to be $35.6 \%$ and $28.1 \%$ for the $\mathrm{TEM}_{5,0}$ mode and $\mathrm{TEM}_{0,0}$ mode, respectively.

Acknowledgements The authors thank the National Science Council for their financial support of this research under Contract No. NSC97-2112-M-009-016-MY3.

\section{References}

1. A.V. Hicks, C.X. Wang, G.Y. Wang, Proc. SPIE 5332, 120 (2004)
2. C.X. Wang, G.Y. Wang, A.V. Hicks, D.R. Dudley, H.Y. Pang, N. Hodgson, Proc. SPIE 6100, 610019 (2006)

3. X.P. Yan, Q. Liu, M. Gong, D.S. Wang, X. Fu, Laser Phys. Lett. 6, 93 (2009)

4. B. Li, J. Yao, X. Ding, Q. Sheng, P. Wang, Opt. Commun. 283, 3497 (2010)

5. C.J. Flood, G. Giuliani, H.M. van Driel, Opt. Lett. 15, 215 (1990)

6. R. Oron, S. Blit, N. Davidson, A.A. Friesem, Z. Bomzon, E. Hasman, Appl. Phys. Lett. 77, 3322 (2000)

7. Y.F. Chen, Y.P. Lan, S.C. Wang, Appl. Phys. B 72, 167 (2001)

8. Y.F. Chen, Y.P. Lan, Phys. Rev. A 63, 063807 (2001)

9. A.A. Ishaaya, N. Davidson, G. Machavariani, E. Hasman, A.A. Friesem, IEEE J. Quantum Electron. 39, 74 (2003)

10. T. Moser, M.A. Ahmed, F. Pigeon, O. Parriaux, E. Wyss, Th. Graf, Laser Phys. Lett. 1, 234 (2004)

11. J.F. Bisson, Yu. Senatsky, K.I. Ueda, Laser Phys. Lett. 2, 327 (2005)

12. M.A. Porras, R. Borghi, M. Santarsiero, J. Opt. Soc. Am. A 18, 177 (2001)

13. J. Durnin, J.J. Miceli, Jr., J.H. Eberly, Phys. Rev. Lett. 58, 1499 (1987)

14. A. Hakola, A. Shevchenko, S.C. Buchter, M. Kaivola, N.V. Tabiryan, J. Opt. Soc. Am. B 23, 637 (2006)

15. A. Hakola, T. Hakkarainen, R. Tommila, T. Kajava, J. Opt. Soc. Am. B 27, 2342 (2010)

16. A.N. Khilo, E.G. Katranji, A.A. Ryzhevich, J. Opt. Soc. Am. A 18, 1986 (2001)

17. F. Wu, Y. Chen, D. Guo, Appl. Opt. 46, 4943 (2007)

18. Y. Matsuoka, Y. Kizuka, T. Inoue, Appl. Phys. A 84, 423 (2006)

19. F. Courvoisier, P.-A. Lacourt, M. Jacquot, M.K. Bhuyan, L. Furfaro, J.M. Dudley, Opt. Lett. 34, 3163 (2009)

20. T. Wulle, S. Herminghaus, Phys. Rev. Lett. 70, 1401 (1993)

21. C. Altucci, R. Bruzzese, D. D’Antuoni, C. de Lisio, S. Solimeno, J. Opt. Soc. Am. B 17, 34 (2000)

22. S. Orlov, A. Stabinis, V. Smilgevičius, G. Valiulis, A. Piskarskas, Opt. Lett. 32, 68 (2007)

23. W. Koechner, Solid-State Laser Engineering, 6th edn. (Springer, Berlin, 2005) 